\title{
$\beta$-elemene induced anticancer effect in bladder cancer through upregulation of PTEN and suppression of AKT phosphorylation
}

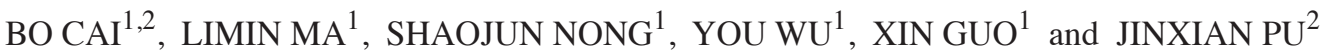 \\ ${ }^{1}$ Department of Urology, Affiliated Hospital of Nantong University, Nantong, Jiangsu 226001; \\ ${ }^{2}$ Department of Urology, The First Affiliated Hospital of Suzhou University, Suzhou, Jiangsu 215001, P.R. China
}

Received August 16, 2017; Accepted August 29, 2018

DOI: $10.3892 / \mathrm{ol} .2018 .9401$

\begin{abstract}
Human bladder cancer is one of the most aggressive tumours known and has shown resistance to traditional chemotherapy, which depends heavily on DNA-damaging drugs. $\beta$-elemene is one of the least cytotoxic antitumor agents that are extracted from Curcuma aromatica salisb and it exhibits antitumor effects in many carcinomas. $\beta$-elemene has attracted the attention of clinicians and scientists worldwide due to its few side effects and limited effect on the bone marrow. However, the antitumor mechanism of $\beta$-elemene remains largely unstudied. In the present study, the expression of the AKT serine/threonine kinase (AKT) signaling pathway in bladder cancer and normal bladder tissue was investigated, and the influence of $\beta$-elemene on bladder cancer cells and the mechanisms involved were assessed. The results showed that phosphatase and tensin homolog deleted on chromosome ten (PTEN) was downregulated and phosphorylated-AKT (pAKT) was overexpressed in human bladder cancer. $\beta$-elemene significantly suppressed the viability of bladder cancer cells, while leaving normal bladder cells unaffected. In addition, there was an increased number of apoptotic bladder cancer cells following $\beta$-elemene treatment, and a significant reduction in cell invasion and migration. Subsequent western blot analyses revealed that bladder cancer cells treated with $\beta$-elemene had increased PTEN expression and decreased expression of pAKT. Taken together, these results suggest that $\beta$-elemene has an antitumor effect in bladder cancer cells through the upregulation of PTEN and suppression of AKT phosphorylation.
\end{abstract}

\section{Introduction}

Human bladder cancer is the one of the most common neoplasms among urological malignancies, and it is a serious public health concern worldwide (1). Chemotherapy, such as

Correspondence to: Professor Jinxian Pu, Department of Urology, The First Affiliated Hospital of Suzhou University, 899 Pinghai Road, Suzhou, Jiangsu 215001, P.R. China

E-mail: sdfyypujinxian@sina.com

Key words: $\beta$-elemene, bladder cancer, PTEN, AKT, apoptosis
Bacillus Calmete-Guerin (BCG) and doxorubicin therapy, is currently the standard treatment regimen for patients suffering from bladder cancer $(2,3)$. Bladder cancer is an aggressive tumor which is always resistant to chemotherapy, and the current chemotherapeutic drugs are still not safe enough for patients with bladder cancer because of their toxicity to normal bladder tissue. Thus, the overall survival rate remains poor because of recurrence and metastases despite the use of multimodal therapy (4).

$\beta$-Elemene ( $\beta$-1-1 methyl-1-vinyl-2,4-di-isopropenylcyclohexane) is derived from the essential oil of the Chinese traditional herbal medicine Curcuma aromatica salisb (5). $\beta$-elemene, which is considered to be a national class II noncytotoxic antitumor agent in China (6), has been shown to have a variety of pharmacological effects on multiple types of malignant cells (7). Moreover, it can enhance the immunogenicity of tumor cells and improve systematic immunity (8). $\beta$-Elemene has drawn a great deal of attention because of its small number of side effects and its bone marrow protection properties (9).

Apoptosis is one of the most important mechanisms that regulates biological processes, and dysregulation of apoptosis is associated with a variety of human diseases such as cancer, infections, and neurodegenerative diseases (10). Apoptosis is the most common and important mechanism initiated by current chemotherapeutic compounds. The AKT pathway is implicated to be one of the most important pathways for cell survival and inhibition of apoptosis. AKT regulates the process of cell survival by phosphorylating different substrates that directly or indirectly regulate the apoptotic program, It has been shown that AKT could regulate various cellular processes, such as cell apoptosis, survival and angiogenesis (11). The inactivation of phosphorylated-AKT (pAKT) has been reported to induce tumor cell apoptosis (12). Phosphatase and tensin homolog deleted on chromosome ten (PTEN) is a tumour suppressor gene that has been shown to be inactivated or to exhibit some level of functional abnormality in various cancers including glioma, prostate carcinoma and renal carcinoma (13). PTEN can inhibit the expression of pAKT which plays an extremely important role in regulating the AKT pathway (14). and its inactivation results in the up-regulation of pAKT (15). Moreover, the inactivation of pAKT has been reported to be one of the key mechanisms leading to apoptosis induced by a number of antitumor drugs (16). 
According to the description given above, we hypothesized that $\beta$-elemene induces its antitumor effect through the AKT pathway. To investigate the mechanism, we first detected the expression of PTEN and pAKT in human bladder cancer and normal tissues and then observed the antitumor effects of $\beta$-elemene on bladder cell proliferation, apoptosis, invasion and migration. Finally, we verify whether $\beta$-elemene has antitumor effects via regulation of the PTEN-AKT pathway.

\section{Materials and methods}

Materials. $\beta$-elemene was obtained from Huali JinGang Pharmaceutical Co., Ltd. (Dalian, China). Human bladder cancer T24 and 5637 cells were purchased from the cell library of the Chinese Academy of Sciences (Shanghai, China), and normal bladder SV-HUC-1 cells from Saibaikang Biotechnology Co., Ltd. (Shanghai, China). Antibodies against PTEN, pAKT, AKT, glyceraldehyde-3-phosphate dehydrogenase (GAPDH), and peroxidase-labelled anti-rabbit IgG were purchased from Abcam (Cambridge, UK). The fetal bovine serum (FBS), Roswell Park Memorial Institute-1640 (RPMI-1640) and Dulbecco's modified Eagle's medium (DMEM) were purchased from Gibco (Thermo Fisher Scientific, Inc., Waltham, MA, USA).

Cell culture. The primary bladder cancer (PBC) cells were derived from the human bladder cancer tissues of the patients in the Department of Urology, Affiliated Hospital of Nantong University (Nantong, China). All of the tissues obtained from patients were approved by the ethics committee of the Affiliated Hospital of Nantong University and written informed consent was obtained from all patients. The human bladder cancer T24 cells, 5637 cells, and PBC cells were propagated in DMEM nutrient mixture supplemented with 10\% FBS, and SV-HUC-1 cells were propagated in RPMI-1640 with $10 \%$ FBS. All of the cell lines were cultured at a $37^{\circ} \mathrm{C}$ humidified atmosphere with $5 \% \mathrm{CO}_{2}$ and $95 \%$ air. We replaced the culture medium with fresh DMEM or RPMI-1640 that contain 10\% FBS after $24 \mathrm{~h}$. Since then, each cell lines were cultured following routine laboratory procedure for subsequent experiments.

Cell survival assay. The cytotoxic effects of $\beta$-elemene on the T24, 5637, PBC and SV-HUC-1 cells were detected using 3-(4,5-dimethylthiazol-2-yl)-2,5-diphenyltetrazolium bromide (MTT) assay. In brief, T24 and PBC cells were seeded in 96-well plates (Corning Incorporated, Corning, NY, USA) at a density of $1 \times 10^{4}$ cells/well with fresh complete culture medium for $24 \mathrm{~h}$. Then, both groups of cells were treated with $\beta$-elemene at a concentration of $0,10,20,50,100,200$ or $500 \mu \mathrm{g} / \mathrm{ml}$ and incubated for 24 and $48 \mathrm{~h}$. The growth of both cell lines was determined by adding $10 \mu 1$ MTT to each well. After incubation for $4 \mathrm{~h}, 150 \mu \mathrm{l}$ dimethyl sulphoxide (DMSO) was added to each well and shaken to dissolve the crystal. The absorbance was read at a wavelength of $490 \mathrm{~nm}$ in a microplate reader (BioRad Laboratories, Inc., Hercules, CA, USA). The cell survival percentage was calculated using the following formula: cell survival $(\%)=100 \% \mathrm{x}$ experimental well/untreated control well.

Assessment of apoptosis. Apoptosis was detected in both cell lines using the Annexin V assay. After T24, 5637 and PBC cells were treated with $43 \mu \mathrm{g} / \mathrm{ml} \beta$-elemene or saline control for $24 \mathrm{~h}$. Both cell lines were harvested with $0.25 \%$ trypsin-ethylenediaminetetraacetic acid (EDTA) and washed with PBS. Then, $5 \mu \mathrm{l}$ of Annexin V-PE and 7-AAD (Sheng Nuokang Biotechnology Company, Jiangsu, China) was added to each well for $15 \mathrm{~min}$ at room temperature according to the manufacturer's instructions. The cell concentration was $1 \times 10^{6}$ cells $/ \mathrm{ml}$. Apoptotic cell death was measured using Annexin V-PE and 7-AAD double staining with a FACSCalibur Flow Cytometer and Cellquest software (BD Biosciences, Franklin Lakes, NJ, USA).

Protein extraction and western blot analysis. Fresh bladder cancer tissue was washed with ice-cold phosphate-buffered solution (PBS) and cut into small pieces then $1 \mathrm{ml}$ RIPA Lysis Buffer and $10 \mu \mathrm{l}$ phenylmethylsulfonyl fluoride (PMSF) were added. After incubation for $30 \mathrm{~min}$, the cellular extracts were clarified by centrifugation at $12,000 \times \mathrm{g}$ at $4^{\circ} \mathrm{C}$ for $20 \mathrm{~min}$. T24 and 5637 cells were harvested after $\beta$-elemene treatment at a concentration of $0-43 \mu \mathrm{g} / \mathrm{ml}$ for $48 \mathrm{~h}$ and then washed in PBS. Next, $200 \mu 1$ RIPA Lysis Buffer and $2 \mu 1$ PMSF were added, and cells were lysed on ice for $20 \mathrm{~min}$. Supernatant fluids were collected after centrifugation for $15 \mathrm{~min}$. Both protein concentrations were measured using the Bradford assay. Protein lysates were separated by electrophoresis on $10 \%$ SDS-polyacrylamide gel and transferred to a PVDF membrane (1:2,000; Beyotime Institute of Biotechnology, Haimen, China). After soaking in blocking buffer for $2 \mathrm{~h}$, the membranes were incubated with PTEN (1:500, 32199; Abcam, Cambridge, MA, USA) and AKT (1:500, 64148, 38449; Abcam) monoclonal antibody overnight at $4^{\circ} \mathrm{C}$. Next, we incubated with an HRP-conjugated secondary antibody $\left(1: 1,000,21058\right.$; Abcam) at $37^{\circ} \mathrm{C}$ for $1 \mathrm{~h}$. All Western bands were quantified using ECL detection (Eastman Kodak, Rochester, NY, USA), and the results were visualized by Image J (National Institutes of Health, Bethesda, MD, USA). GAPDH was detected as a gel loading control. All western bands were quantified using densitometry and presented in the form of bar graphs.

In vitro cell invasion and migration assays. 24-well Transwell plates with $8-\mu \mathrm{m}$ polycarbonate filters were used for the cell invasion assay. T24 and 5637 bladder cells were seeded in serum-free DMEM with or without $\beta$-elemene $(0-43 \mu \mathrm{g} / \mathrm{ml})$ with a membrane coated with Matrigel basement membrane matrix $\left(100 \mu \mathrm{g} / \mathrm{cm}^{2}\right)$. DMEM with $10 \%$ serum was added to the bottom of the plates. To observe the changes of motility better, after $48 \mathrm{~h}$ (17), the cells that had not invaded through the membrane were removed, and the invasive cells on the lower surface were fixed in $4 \%$ paraformaldehyde for $20 \mathrm{~min}$ before staining with crystal violet $(0.1 \%)$ for $10 \mathrm{~min}$. The cells that invaded to the lower side were counted with an Olympus IX71 microscope (x100). In the wound healing assay, T24 bladder cells were cultured in 6-well plates and a scratch was made in the monoculture using a $100-\mu 1$ pipette tip. Three reference marks were made in each well. The distances were measured at 0 and $48 \mathrm{~h}$ post-wounding by light-phase photographs (x40).

Statistical data analysis. All experiments were repeated at least three times. Data are presented as the mean \pm standard deviation (SD). The Student's t-test and one-way ANOVA 

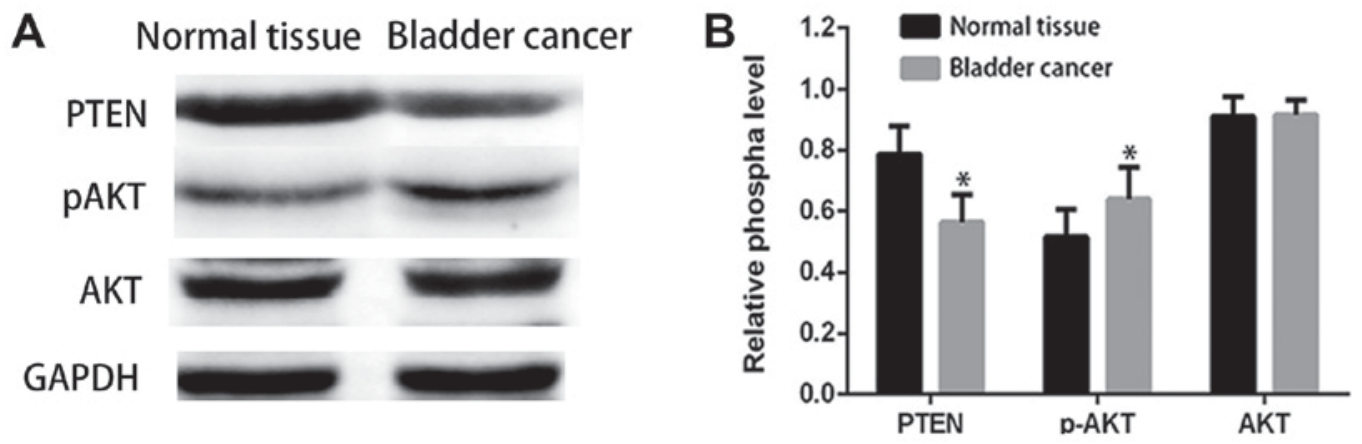

Figure 1. (A) Western blot analysis. (B) PTEN was down-regulated and pAKT was over-expressed in human bladder cancer according to the results of WB. ${ }^{*} \mathrm{P}<0.05$ vs. normal tissue.
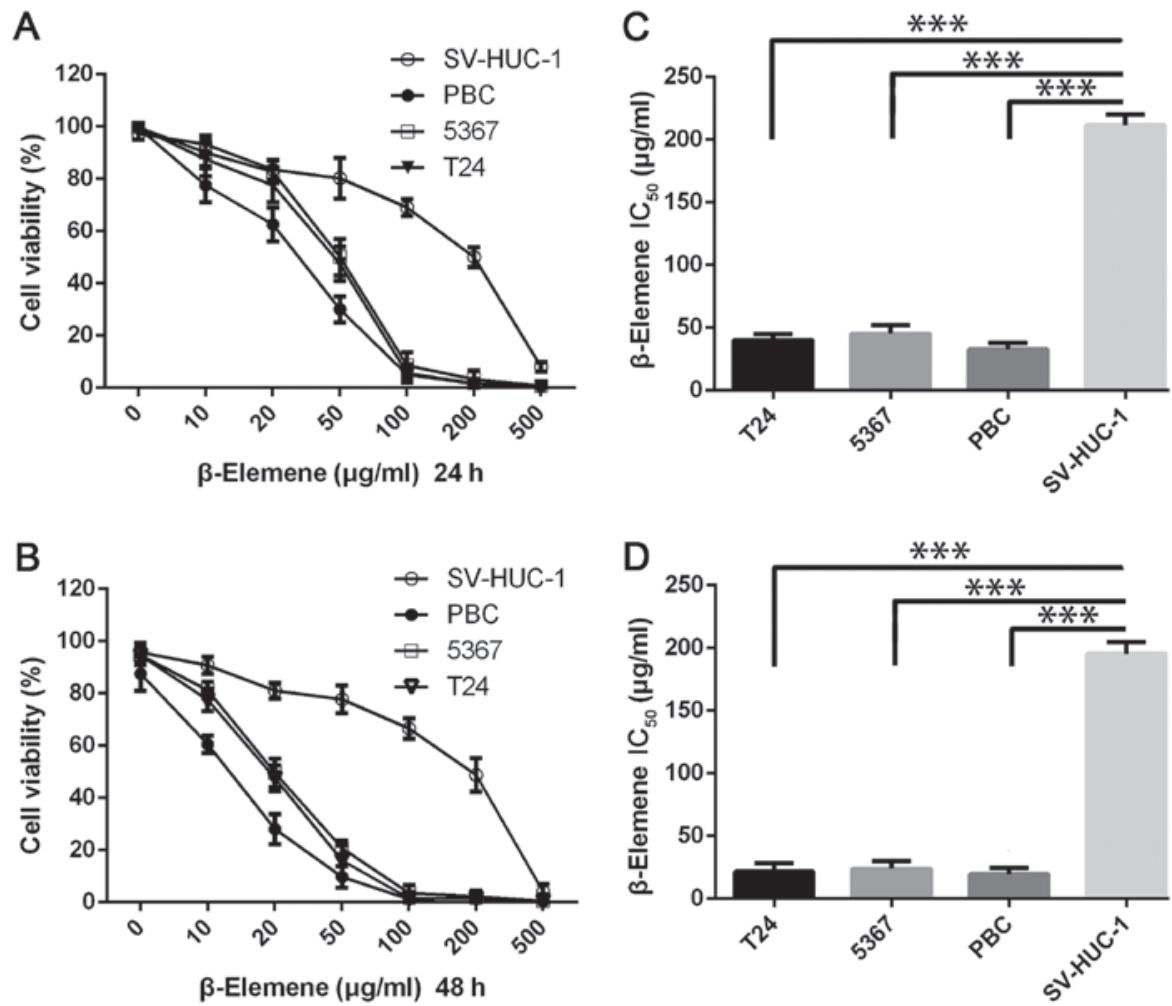

Figure 2. Cytotoxicity of $\beta$-elemene towards three bladder cancer cell lines (T24, 5637, and PBC) and one normal bladder cell line (SV-HUC-1); the exponentially growing cells were treated with $\beta$-elemene for (A) $24 \mathrm{~h}$ and (B) $48 \mathrm{~h}$. The average half-maximal inhibitory concentration (IC $\mathrm{IC}_{50}$ ) values of all the cell lines. (C) $24 \mathrm{~h}$. (D) $48 \mathrm{~h}$. The $\mathrm{IC}_{50}$ values of $\beta$-elemene on SV-HUC-1 were much higher than these three bladder cancer cells. ${ }^{* * *} \mathrm{P}<0.001$. PBC, primary bladder cancer.

were used to determine the statistical significance between the different experimental groups and the control group. Student-Newman-Keuls test was used as post hoc test after ANOVA. All data were analysed using SPSS 20.0 (IBM Corp., Armonk, NY, USA) and GraphPad prism software (v.6; GraphPad Software, Inc., La Jolla, CA, USA). P $<0.05$ was considered to indicate a statistically significant difference.

\section{Results}

PTEN is downregulated and $p A K T$ is over-expressed in human bladder cancer. To determine the role of PTEN and AKT in bladder cancer progression, we used Western blot analysis to examine PTEN and AKT expression levels in bladder cancer tissues and compared them to normal tissues. The results shown in Fig. 1A and B indicate results revealed that PTEN protein levels were down-regulated in bladder cancers relative to adjacent normal tissues, while the pAKT level was overexpressed. These results indicate that the decreased PTEN expression and increased pAKT expression may be related to bladder cancer progression.

$\beta$-elemene suppresses the survival of bladder cancer cells. The human bladder cancer cells T24, 5637, PBC cell lines and normal human bladder cell line SV-HUC-1 were used to investigate the effect of $\beta$-elemene on bladder cancer cell growth and proliferation using the MTT assay. As shown in Fig. $2 \mathrm{~A}$ and B, $\beta$-elemene inhibited the survival of the T24 and 5637 bladder cancer cell lines, and the magnitude of inhibition increased in a dose-dependent manner. To further assess the 
A Control
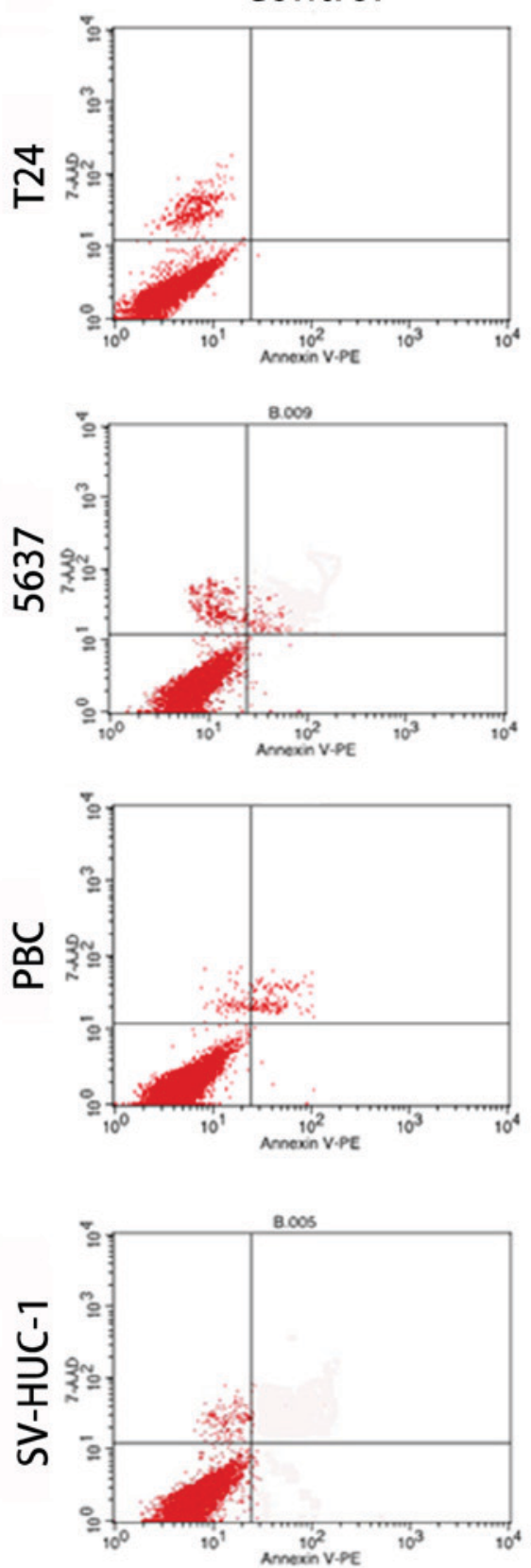

$\beta$-elemene
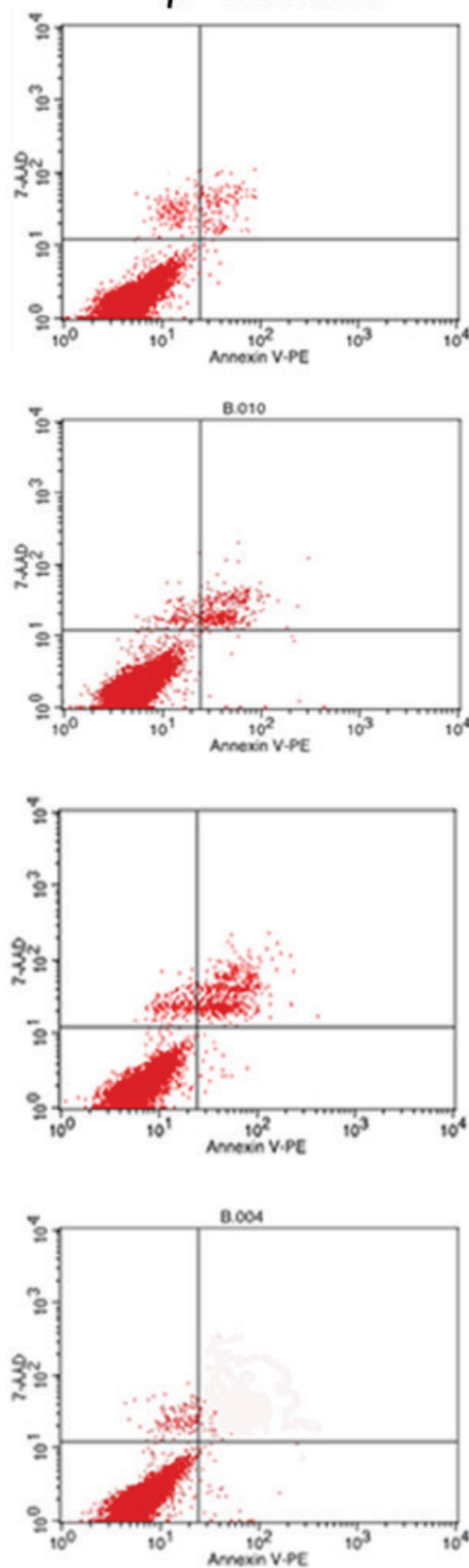

B
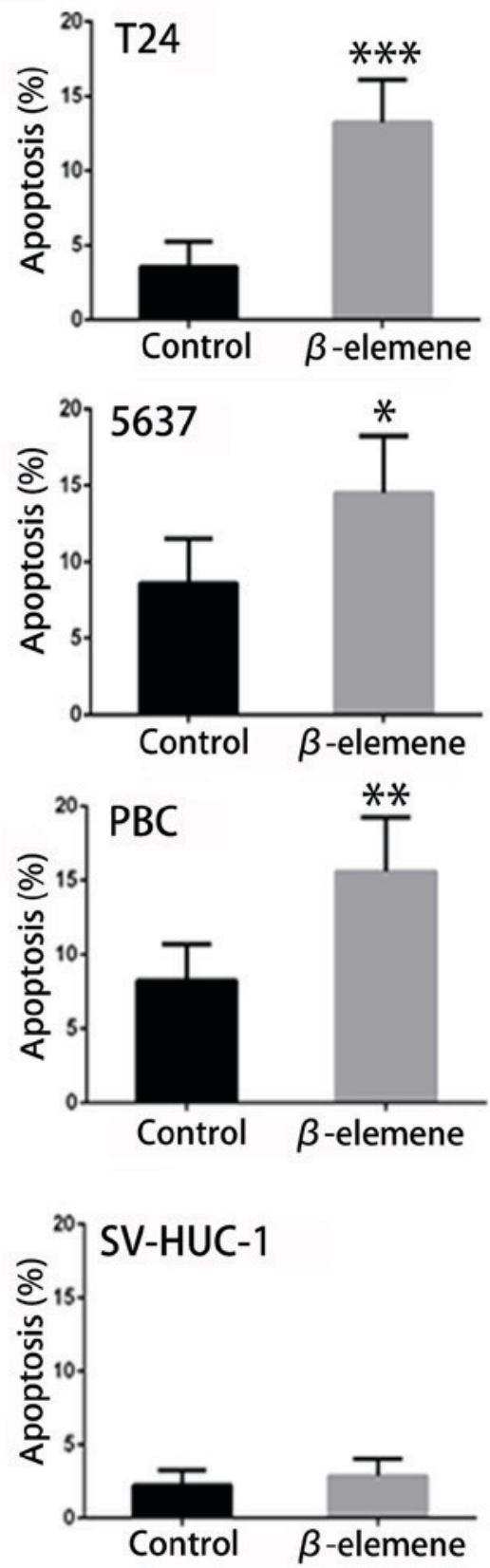

Figure 3. (A) Detection of $\beta$-elemene-induced apoptosis in the three human bladder cancer cell lines and one normal bladder cell line by flow cytometry. (B) The percentage of apoptotic cells increased significantly in the bladder cancer cells treated with $\beta$-elemene compare with the normal bladder cells. "P<0.05, ${ }^{* *} \mathrm{P}<0.01,{ }^{* * *} \mathrm{P}<0.001$.

anti-cancer activity of $\beta$-elemene on bladder cancer, we used primary cancer cells (PBC) derived from patient with bladder cancer, and the results were same as the former two bladder cancer cells. $\beta$-Elemene exerted dramatic cytotoxicity on the three bladder cancer cell lines at $10 \mu \mathrm{g} / \mathrm{ml}$ in $48 \mathrm{~h}$, and the inhibitory rate was $>90 \%$ at a concentration of $100 \mu \mathrm{g} / \mathrm{ml}$ or higher in both 24 and 48 h. However, no matter 24 or 48 h, the results revealed no significant sensitivity to $\beta$-elemene in normal human bladder cells SV-HUC-1. As shown in Fig. 2C and D, the average half-maximal inhibitory concentration $\left(\mathrm{IC}_{50}\right)$ of $\beta$-elemene was $43 \pm 5.9 \mu \mathrm{g} / \mathrm{ml}$ for T2 4 cells, $47 \pm 7.1 \mu \mathrm{g} / \mathrm{ml}$ for 5,367 cells and $33.5 \pm 5.9 \mu \mathrm{g} / \mathrm{ml}$ for PBC cells at $24 \mathrm{~h}$, and the magnitude of the $\mathrm{IC}_{50}$ decreased at $48 \mathrm{~h}$ was
21.5 $\pm 7.6 \mu \mathrm{g} / \mathrm{ml}$ for T24 cells, $23.75 \pm 6.3 \mu \mathrm{g} / \mathrm{ml}$ for 5,367 cells and $19.5 \pm 5.5 \mu \mathrm{g} / \mathrm{ml}$ for PBC cells. According to the data from MTT assay, the $\mathrm{IC}_{50}$ values of $\beta$-elemene on SV-HUC-1 were much higher than these three bladder cancer cells in both 24 and $48 \mathrm{~h}$. These data showed that $\beta$-elemene effectively inhibits the growth and proliferation of bladder cancer cells without significant cytotoxicity to normal cells.

$\beta$-elemene induces apoptosis in human bladder cancer cells. To investigate the mechanism of $\beta$-elemene's inhibitory activity on the growth of bladder cancer, we first performed flow cytometry to detect apoptotic cells based on Annexin V staining on the outer cytoplasmic membrane to identify apoptotic cells. As 
A

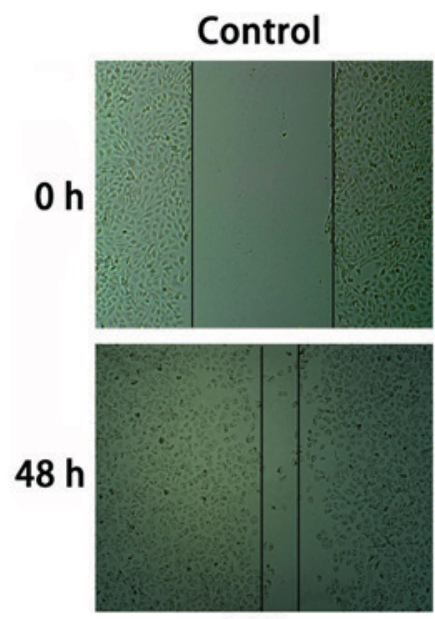

T24
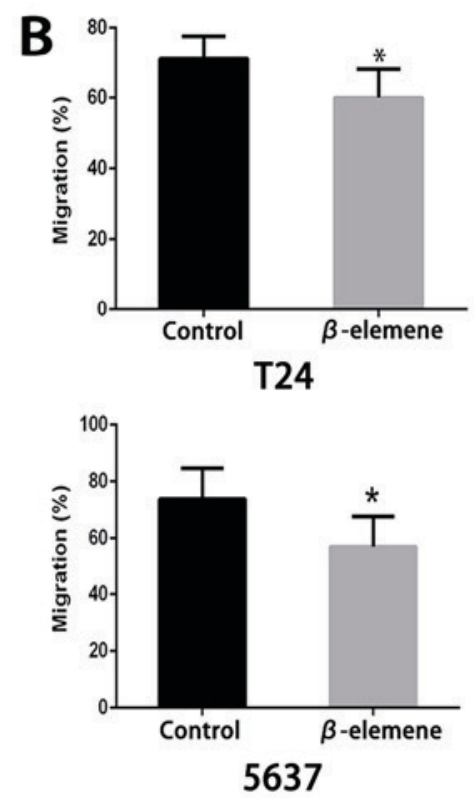

$\beta$-elemene
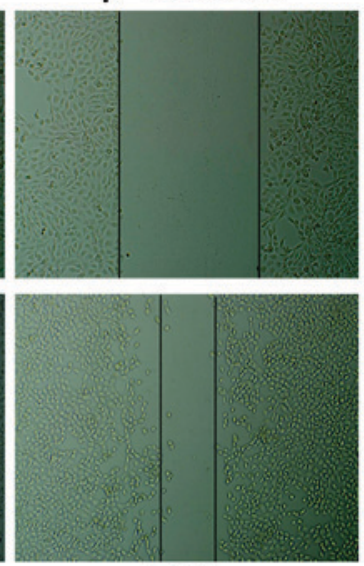

T24

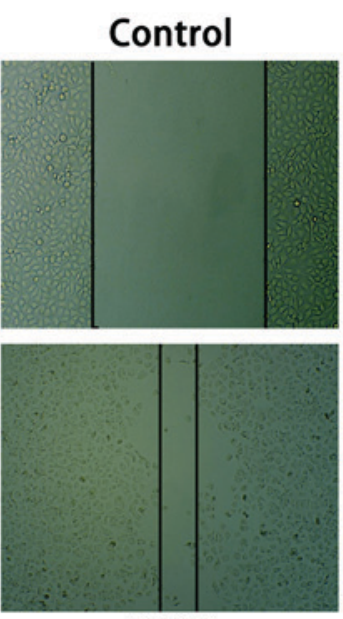

5637

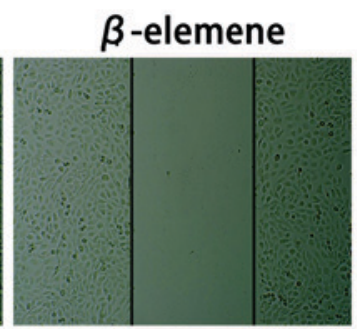

C
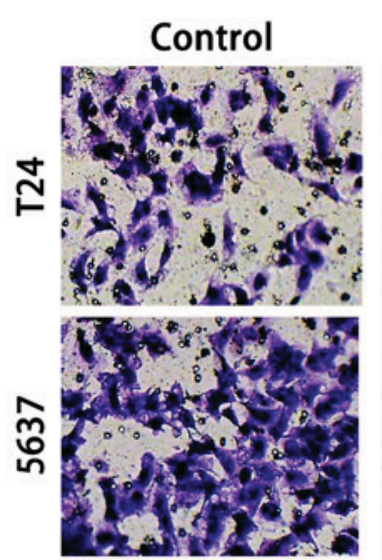

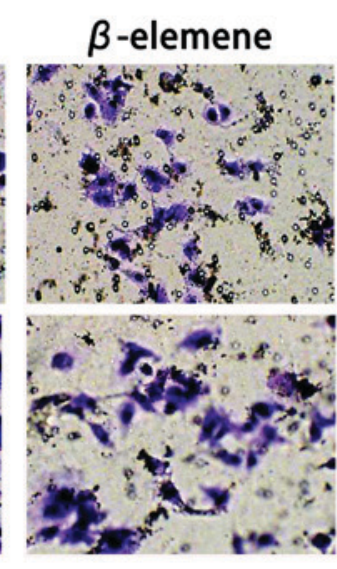

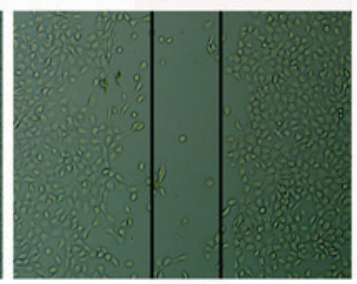

5637

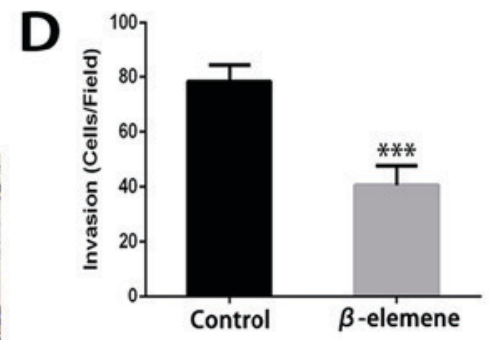

T24

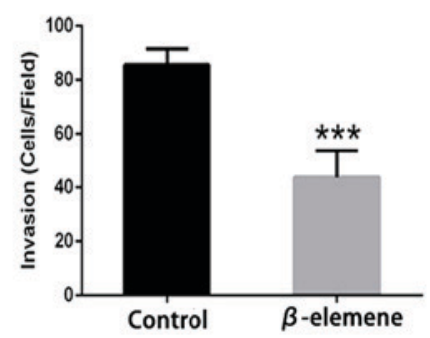

5637

Figure 4. Images of T24 and 5637 bladder cancer cells traversed the Matrigel basement membrane after incubation with $\beta$-elemene (A) and corresponding quantitative results (B). Images of T24 and 5637 bladder cancer cells migrated for $48 \mathrm{~h}$ after affected by $\beta$-elemene (C) (magnification, x100) and corresponding results (D). ${ }^{*} \mathrm{P}<0.05,{ }^{* * *} \mathrm{P}<0.001$.

shown in Fig. 3A and B, the ratio of apoptosis was significantly higher in T24, 5637 and PBC cells treated with $\beta$-elemene than in untreated cells in $24 \mathrm{~h}$. In contrast, no significant changes were observed in SV-HUC-1 cells. These results indicated that $\beta$-elemene induced apoptosis in bladder cancer cells, but not in normal bladder cells.

$\beta$-elemene changes the motility of bladder cancer cells. To further study the changes of mobility in bladder cancer cells after $\beta$-elemene treatment, we used the wound healing assay and transwell assay to detect cell migration and changes in invasion. As shown in Fig. 4A and B, in both types of bladder cancer cells, $\beta$-elemene (approximately $10 \mu \mathrm{g} / \mathrm{ml}$ ) could significantly restrain bladder cancer cells migration compared with the controls. In the Matrigel invasion assay, the percentage of invading T24 and 5637 cells significantly decreased after $\beta$-elemene treatment (Fig. 4C and D). These results suggested that $\beta$-elemene could negatively regulate bladder cancer cell motility by weakening bladder cancer cell migration and invasion. $\beta$-elemene enhances PTEN and inhibits pAKT activation. To further investigate the mechanism underlying $\beta$-elemene's anticancer effect, we performed Western blot analysis to detect changes in AKT pathway activation, which plays an important role in regulating cell survival and apoptosis. As shown in Fig. 5A and B, AKT phosphorylation was greatly decreased in $\beta$-elemene-treated T24 and 5637 cells compared with both control groups in $24 \mathrm{~h}$. Moreover, there was significantly more PTEN expression than in controls. However, there was no effect on the expression of total AKT in both bladder cancer cells. The schematic of the study design is shown in Fig. 5C. These results indicated that $\beta$-elemene induced an anticancer effect in bladder cancer through upregulation of PTEN and suppression of AKT phosphorylation.

\section{Discussion}

The development of bladder cancer is complicated, involving multiple genes, processes and stages. Abnormality in 
A

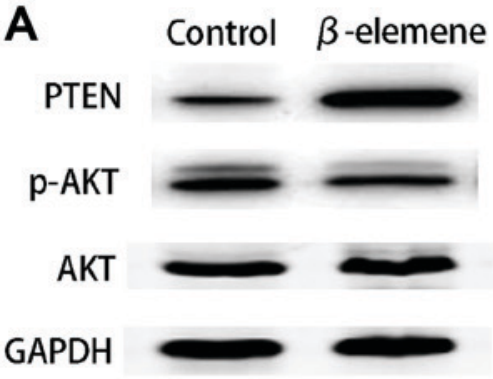

T24

B

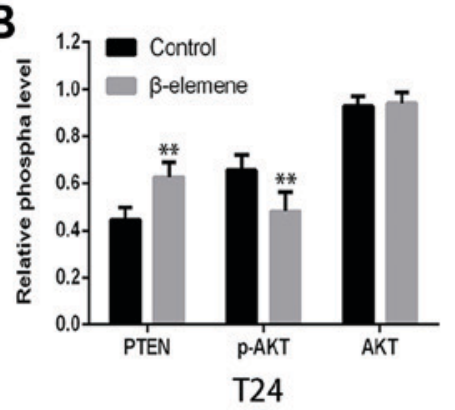

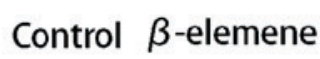

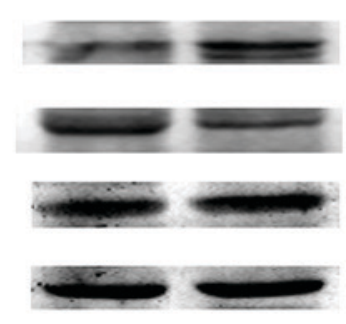

5367

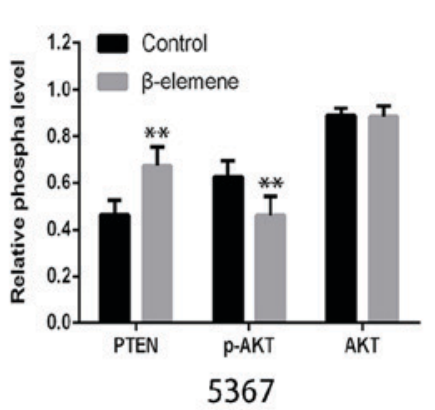

C

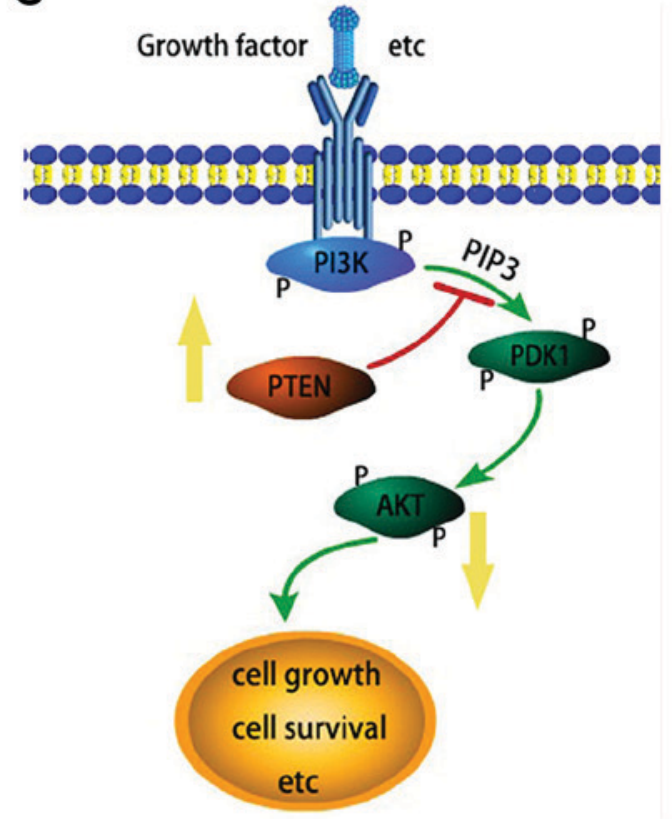

Figure 5. (A) Western blot analysis for PTEN, AKT and pAKT affected by $\beta$-elemene in T24 and 5637 bladder cancer cells and (B) corresponding quantitative results. (C) Schematic of the study design. ${ }^{* *} \mathrm{P}<0.01$. PTEN, phosphatase and tensin homolog deleted on chromosome ten; AKT, AKT serine/threonine kinase; pAKT, phosphorylated-AKT.

apoptosis correlates is closely correlated with bladder cancer progression. Chemotherapeutic agents often kill tumor cells by promoting apoptosis, and the AKT signalling pathway has been shown to be one of the most important pathways for tumor cell proliferation and apoptosis (18). PTEN appears to regulate the AKT signalling pathway by inhibiting the activation of AKT, leading to the inhibition of bladder cancer cell growth in vitro (15). In this study, we found PTEN protein levels to be down-regulated and pAKT to be over-expressed in bladder cancers relative to adjacent normal tissues, which indicate that the decreased PTEN expression and increased pAKT expression may be related to bladder cancer progression.

$\beta$-elemene, a natural active element extracted from Curcuma aromatica salisb, has many diverse functions (19). An emulsion form of $\beta$-elemene has been applied as a class II noncytotoxic antitumor agent in China (20). The major advantages of $\beta$-elemene as an anticancer agent are that it has antitumor activity toward a broad spectrum of cancer types, including lung cancer, brain tumors and malignancies of the alimentary tract, and it is associated with a low level of toxicity which is well-tolerated by patients with cancer (21-23). Consistent with other reports, this study showed that $\beta$-elemene exhibits high levels of anticancer activity on T24, 5637 and PBC bladder cancer cells by suppressing survival and promoting apoptosis compared with SV-HUC-1 normal bladder cells. In addition, we also found $\beta$-elemene could weaken the bladder cancer cells migration and invasion.

However, the mechanism by which $\beta$-elemene inhibits tumors is not fully clear although it has been studied for years. The ability of $\beta$-elemene to regulate signaling pathways has attracted much attention recently (24). The TGF- $\beta$ pathway and AKT pathway have drawn special attention (18). Lu et al reported that $\beta$-elemene could inhibit the proliferation of T24 bladder cancer cells through up-regulation of the expression of Smad4 (25). It has also been demonstrated that the inhibition of pAKT can induce tumor cell apoptosis, pAKT and its downstream targets are thought to be associated with the development of drug resistance (14). The AKT pathway is frequently activated in human bladder cancer cells, and activation of AKT is associated with anti-apoptosis, cell proliferation and cellular energy metabolism effects. Tanaka and Grossman found that PTEN gene therapy can suppress bladder cancer cell growth by down-regulating pAKT (26). For this reason, inhibition of the AKT pathway may be as a promising strategy for cancer treatment, because apoptosis induction is one of the major mechanisms by which natural compounds, such as curcumin and chorophyllin, exert their anti-tumor activity on bladder cancer cells. Lin et al demonstrated that baicalin induced apoptotic cell death through inhibition of the AKT signal pathway in human bladder cancer cells (27). And Zhao et al observed that altholactone down-regulates the expression of the anti-apoptotic protein pAKT in T24 cells (28).

In this work, further experiments showed that the inactivation of the AKT pathway is closely associated with the effect of $\beta$-elemene on bladder cancer cells. The data gathered from T24 and 5637 bladder cancer cells with treated $\beta$-elemene revealed that the expression of PTEN was increased, and AKT phosphorylation was suppressed. Taken together, Theses promising results suggested that $\beta$-elemene induced anticancer effects in bladder cancer occurred through up-regulation of PTEN and suppression of AKT phosphorylation. However further investigation is needed to validate how $\beta$-elemene leads to inactivation of AKT.

\section{Acknowledgements}

Not applicable. 


\section{Funding}

This work was supported by the National Natural Science Foundation of China (grant no. 81141056).

\section{Availability of data and materials}

The analyzed data sets generated during the study are available from the corresponding author on reasonable request.

\section{Authors' contributions}

$\mathrm{BC}$ conceived and designed the experiments, and contributed to the cell culture, cell survival assay, assessment of apoptosis and manuscript preparation. LM contributed to the collection data and statistical analysis in this work. SN, YW and XG contributed to purchase reagents and materials, protein extraction, Western blot analysis, cell invasion and migration assays. JP contributed to the conception, design and suggestion of the study, approved the final version and other routine work.

\section{Ethics approval and consent to participate}

This work was approved by the Ethics Committee of the Affiliated Hospital of Nantong University. Written informed consent was obtained from patients.

\section{Patient consent for publication}

Patients consented to the publication of this work.

\section{Competing interests}

The authors declare that they have no competing interests.

\section{References}

1. Kaprin AD, Kostin AA, Rapoport LM, Tsarichenko DG, Bernikov AN, Vorob'ev NV and Golovashchenko MP: Cancer of urinary bladder. Urologiia 6 (Suppl): S64-S88, 2016 (In Russian).

2. Kamat AM, Hahn NM, Efstathiou JA, Lerner SP, Malmstrom PU, Choi W, Guo CC, Lotan Y and Kassouf W: Bladder cancer. Lancet 388: 2796-2810, 2016.

3. Lan Y, Liu D and Lin M: Comparison of the combination therapy of bacillus Calmette-Guerin and mitomycin $\mathrm{C}$ with the monotherapy for non-muscle-invasive bladder cancer: A meta-analysis. Neoplasma 63: 967-976, 2016.

4. Krasnow RE, Drumm M, Roberts HJ, Niemierko A, Wu CL, Wu S, Zhang J, Heney NM, Wszolek MF, Blute ML, et al: Clinical outcomes of patients with histologic variants of urothelial cancer treated with trimodality bladder-sparing therapy. Eur Urol 72: 54-60, 2017.

5. Ding XF, Shen M, Xu LY, Dong JH and Chen G: 13,14-bis(cis-3, 5 -dimethyl-1-piperazinyl)- $\beta$-elemene, a novel $\beta$-elemene derivative, shows potent antitumor activities via inhibition of mTOR in human breast cancer cells. Oncol Lett 5: 1554-1558, 2013.

6. Hu Z, Wu H, Li Y, Hou Q, Wang Y, Li S, Xia B and Wu S: $\beta$-Elemene inhibits the proliferation of esophageal squamous cell carcinoma by regulating long noncoding RNA-mediated inhibition of hTERT expression. Anticancer Drugs 26: 531-539, 2015.

7. Zou S, Wang C, Cui Z, Guo P, Meng Q, Shi X, Gao Y, Yang G and Han Z: $\beta$-Elemene induces apoptosis of human rheumatoid arthritis fibroblast-like synoviocytes via reactive oxygen species-dependent activation of p38 mitogen-activated protein kinase. Pharmacol Rep 68: 7-11, 2016.
8. Chen JC, Duan WL, Bai RR, Yao HQ, Wu XM, Shang J and Xu JY: Synthesis of $13-\beta$-elemene ester derivatives and evaluation of their antioxidant activity in human umbilical vein endothelial cells. Chin J Nat Med 13: 618-627, 2015.

9. Hong L, Zeng Y and Yang D: Inhibitory effect of $\beta$-elemene on human airway granulation tissue in vivo and in vitro. Respiration 92: 329-338, 2016.

10. Bai H, Li H, Li W, Gui T, Yang J, Cao D and Shen K: The $\mathrm{PI} 3 \mathrm{~K} / \mathrm{AKT} / \mathrm{mTOR}$ pathway is a potential predictor of distinct invasive and migratory capacities in human ovarian cancer cell lines. Oncotarget 6: 25520-25532, 2015.

11. Olasz J, Doleschall Z, Dunai Z, Pazsitka A and Csuka O: PI3K/AKT pathway activation and therapeutic consequences in breast cancer. Magy Onkol 59: 346-351, 2015 (In Hungarian)

12. Brotelle T and Bay JO: PI3K-AKT-mTOR pathway: Description, therapeutic development, resistance, predictive/prognostic biomarkers and therapeutic applications for cancer. Bull Cancer 103: 18-29, 2016 (In French).

13. Rana C, Piplani H, Vaish V, Nehru B and Sanyal SN: Downregulation of PI3-K/Akt/PTEN pathway and activation of mitochondrial intrinsic apoptosis by Diclofenac and Curcumin in colon cancer. Mol Cell Biochem 402: 225-241, 2015.

14. Chun SH, Jung CK, Won HS, Kang JH, Kim YS and Kim MS: Divergence of P53, PTEN, PI3K, Akt and mTOR expression in tonsillar cancer. Head Neck 37: 636-643, 2015.

15. Lim HJ, Crowe P and Yang JL: Current clinical regulation of $\mathrm{PI} 3 \mathrm{~K} / \mathrm{PTEN} / \mathrm{Akt} / \mathrm{mTOR}$ signalling in treatment of human cancer. J Cancer Res Clin Oncol 141: 671-689, 2015.

16. Chin CC, Li JM, Lee KF, Huang YC, Wang KC, Lai HC, Cheng CC, Kuo YH and Shi CS: Selective $\beta 2$-AR blockage suppresses colorectal cancer growth through regulation of EGFR-Akt/ERK1/2 signaling, G1-phase arrest, and apoptosis. J Cell Physiol 231: 459-472, 2016.

17. Zhu Y, Dai B, Zhang H, Shi G, Shen Y and Ye D: Long non-coding RNA LOC572558 inhibits bladder cancer cell proliferation and tumor growth by regulating the AKT-MDM2-p53 signaling axis. Cancer Lett 380: 369-374, 2016.

18. Chen J, Wang T, Xu S, Zhang P, Lin A, Wu L, Yao H, Xie W, Zhu Z and $\mathrm{Xu}$ J: Discovery of novel antitumor nitric oxide-donating $\beta$-elemene hybrids through inhibiting the PI3K/Akt pathway. Eur J Med Chem 135: 414-423, 2017.

19. Yu X, Xu M, Li N, Li Z, Li H, Shao S, Zou K and Zou L: $\beta$-elemene inhibits tumor-promoting effect of M2 macrophages in lung cancer. Biochem Biophys Res Commun 490: 514-520, 2017.

20. Mu L, Wang T, Chen Y, Tang X, Yuan Y and Zhao Y: $\beta$-Elemene enhances the efficacy of gefitinib on glioblastoma multiforme cells through the inhibition of the EGFR signaling pathway. Int J Oncol 49: 1427-1436, 2016.

21. Zhang J, Zhang HD, Yao YF, Zhong SL, Zhao JH and Tang JH: $\beta$-elemene reverses chemoresistance of breast cancer cells by reducing resistance transmission via exosomes. Cell Physiol Biochem 36: 2274-2286, 2015.

22. Chen J, Wang R, Wang T, Ding Q, Khalil A, Xu S, Lin A, Yao H, Xie W, Zhu Z and Xu J: Antioxidant properties of novel dimers derived from natural $\beta$-elemene through inhibiting $\mathrm{H}_{2} \mathrm{O}_{2}$-induced apoptosis. ACS Med Chem Lett 8: 443-448, 2017.

23. Ma C, Zhou W, Yan Z, Qu M and Bu X: $\beta$-Elemene treatment of glioblastoma: A single-center retrospective study. Onco Targets Ther 9: 7521-7526, 2016.

24. Chen X, Wang Y, Luo H, Luo Z, Zhang T, Yang N, Long X, Xie H, Qiu W, Zhang B, Ding J and Yang L: $\beta$-elemene acts as an antitumor factor and downregulates the expression of survivin, Bcl-xL and Mta-1. Mol Med Rep 6: 989-995, 2012.

25. Lu X, Wang Y, Luo H, Qiu W, Han H and Chen X: $\beta$-elemene inhibits the proliferation of T24 bladder carcinoma cells through upregulation of the expression of Smad4. Mol Med Rep 7: 513-518, 2013.

26. Tanaka $M$ and Grossman HB: In vivo gene therapy of human bladder cancer with PTEN suppresses tumor growth, downregulates phosphorylated $\mathrm{Akt}$, and increases sensitivity to doxorubicin. Gene Ther 10: 1636-1642, 2003.

27. Lin C, Tsai SC, Tseng MT, Peng SF, Kuo SC, Lin MW, Hsu YM, Lee MR, Amagaya S, Huang WW, et al: AKT serine/threonine protein kinase modulates baicalin-triggered autophagy in human bladder cancer T24 cells. Int J Oncol 42: 993-1000, 2013.

28. Zhao B and Li X: Altholactone induces reactive oxygen species-mediated apoptosis in bladder cancer T24 cells through mitochondrial dysfunction, MAPK-p38 activation and Akt suppression. Oncol Rep 31: 2769-2775, 2014. 